\title{
Eye movements, not hypercompatible mappings, are critical for eliminating the cost of task set reconfiguration
}

\author{
AMELIA R. HUNT \\ University of British Columbia, Vancouver, British Columbia, Canada \\ and \\ YOKO ISHIGAMI and RAYMOND M. KLEIN \\ Dalhousie University, Halifax, Nova Scotia, Canada
}

\begin{abstract}
Residual switch costs are notoriously difficult to eliminate. Yet Hunt and Klein (2002) eliminated them in a task that required observers to alternate between 8 trials of prosaccades and 8 trials of antisaccades, as long as there was at least $1 \mathrm{sec}$ between the task cue and the onset of the saccade target. It was proposed that the elimination of residual switch costs occurred because prosaccade responses are computed very rapidly. These so-called hypercompatible responses bypass memory retrieval stages of the response process, thereby eliminating the source of residual switch costs. Here we tested this hypothesis by requiring observers to alternate between responding with the finger that was vibrated (another task that meets the criteria for hypercompatibility) and responding with the finger of the opposite hand. Residual switch costs were not eliminated, suggesting that their elimination in Hunt and Klein (2002) was due to special properties of the prosaccade-antisaccade task.
\end{abstract}

Performance involving two alternating tasks is significantly worse than performance involving a single task (see, e.g., Allport, Styles, \& Hsieh, 1994). The cost of switching between tasks can be reduced as a function of preparation time, but the switch cost is not completely eliminated, even when ample time to prepare for the impending change in task is provided (Meiran, 1996; Rogers \& Monsell, 1995). Until recently, these "residual switch costs" were considered to be such a fundamental component of task switching that they could not be eliminated (see, e.g., Pashler et al., 2000). According to the widely accepted view, reconfiguration is complete only with the appearance of the target stimulus for the new task (Rogers \& Monsell, 1995). There are, therefore, at least two components of the task-switching process: One is voluntary control of task set reconfiguration, and the other, which gives rise to residual cost, is exogenously driven by the arrival of the imperative stimulus.

Recently, however, we challenged the conventional idea that the residual switch cost is a necessary component of executive control in task switching (Hunt \& Klein, 2002). In this study, participants switched between a prosaccade

The authors thank John Christie and David Shore for technical assistance and the reviewers for their comments. A.R.H. was supported by the Natural Sciences and Engineering Research Council (NSERC) of Canada. Y.I. was supported by a Killam Scholarship. This research was made possible by an NSERC operating grant to R.M.K. Correspondence concerning this article should be addressed to A. R. Hunt, Harvard University Vision Sciences Laboratory, 33 Kirkland Street, 7th Floor, Cambridge, MA 02138 (e-mail: ahunt@wjh.harvard.edu). task (a spatially compatible eye movement) and an antisaccade task (an incompatible eye movement). Residual switch costs were eliminated in both reaction time (RT) and accuracy. In Hunt and Klein (2002), we suggested that four conditions contributed to the elimination of switch costs: (1) a sufficiently long preparatory interval; (2) a predictable target onset time; (3) an easily interpreted task cue; and, (4) most importantly, application of the simplicity, or reflexive nature, of the saccadic response system.

The task applied in our 2002 study was critically different from most other laboratory tasks used in task switching. Conventional tasks, such as the Stroop color-word task, require of the subject additional processing in order to translate the relevant attribute of the stimulus into the arbitrary response according to the experimenter's instructions. In Hunt and Klein (2002), the task was characterized by a hypercompatible stimulus-response (S-R) mapping, for which "the relationship between the stimulus and the response is sufficiently strong to require very little attention or memory to perform" (pp. 537-538). If the residual switch cost were associated with interference from memory retrieval processes, and thus constituted an additional process before response execution (Logan, 1988; Meiran, 2000; Wylie \& Allport, 2000), hypercompatible S-R mappings, dissociated from a memory retrieval stage, would have an advantage, resulting in the elimination of residual costs.

The purpose of the present research was to examine the idea that the use of hypercompatible mappings is responsible for eliminating residual costs. We tested this idea using another hypercompatible task - namely, a tactilemanual response task. As we used it, requiring the partici- 
pant to respond with the finger that is vibrated (or the opposite finger), the two versions of this task are analogous to prosaccades and antisaccades. This kind of tactile-manual response task, like the saccade task, exhibits (1) very small practice effects; (2) little to no dual-task interference (see, e.g., Pashler, Carrier, \& Hoffman, 1993); and (3) no increase in RT with the number of response alternatives (see, e.g., Dassonville, Lewis, Foster, \& Ashe, 1999; Fitts \& Posner, 1967, p. 105; Leonard, 1959). Tactile-manual responses are also similar to saccades in terms of the number of stimulus types and response alternatives. ${ }^{1}$ If residual costs are eliminated, this will support Hunt and Klein's (2002) proposal that residual switch costs can be eliminated by using hypercompatible $\mathrm{S}-\mathrm{R}$ mappings. On the other hand, if residual switch costs are not eliminated, it will be necessary to devise a different explanation for their elimination when saccades are used. As was the case in the experiments of Hunt and Klein, performance (RT and accuracy) was examined as a function of stimulus onset asynchrony (SOA; 200, 550, and 1,100 msec), task (compatible or incompatible), and position (trial after the switch, from Trial 1 to Trial 8). Participants responded to left and right tactile stimuli (vibration) by making either spatially compatible or incompatible finger lift responses.

\section{METHOD}

Each of the 14 participants was seated alone in a darkened room, $57 \mathrm{~cm}$ from a central red/green light-emitting diode (LED). Two levers were fixed to the table in front of each participant, one lever for each hand. Each lever was affixed with a hearing aid, acting as a tactile stimulator, on the top of which the participants rested their left and right index fingers. The hearing aids were adjusted so that the left and right finger stimulations were of perceptually similar intensity, as subjectively reported by each participant. The participants wore headphones playing white noise of sufficient intensity to mask the sound of the stimulators.

The participants completed 480 trials in three equal blocks. Each trial began when the participant pressed down on both levers. At this point, either the green or the red LED was illuminated and it remained on until the participant responded. After 200, 550, or $1,100 \mathrm{msec}$ from the onset of the LED, one of the two hearing aids began to vibrate. Participants were instructed to use the color of the LED to prepare for one of two tasks. If the LED was green, participants were instructed to lift the finger that felt the stimulation (i.e., make a compatible response). If the LED was red, they were instructed to lift the opposite finger (an incompatible response). The hearing aid continued to vibrate until the participant responded. Participants depressed both levers for the next trial to begin. The color of the LED changed between red and green every 8 trials, so that participants made 8 compatible responses, followed by 8 incompatible responses, and so on for the duration of each block. The location of the tactile stimulation (left or right) was randomized. The SOA between the instructional LED and the onset of the tactile stimulation was varied between blocks, and the order of blocks was randomized across individual participants. A 486 PC controlled the LED and tactile stimulators and collected response information from the levers.

The first 16 trials of each block were considered practice and excluded from analysis. In the RT analyses, trials on which an incorrect response was executed and trials with RTs less than $120 \mathrm{msec}$ or greater than $1,280 \mathrm{msec}(2.2 \%$ of trials) were also excluded. Trials were labeled according to their position in the sequence of trials fol- lowing a switch (1 through 8 ), whether they were compatible or incompatible, and whether the SOA between the LED and the vibrator was 200,550 , or $1,100 \mathrm{msec}$. Using these three factors, three-way repeated measures ANOVAs were conducted on RT and accuracy. To obtain a more specific measure of task switch costs, we also compared the mean of Trial 1 in the sequence with the means of Trials 4 , 5,6 , and 7 in each condition. ${ }^{2}$

\section{RESULTS}

\section{Reaction Times}

The results are illustrated in Figure 1. When all eight positions were included in the ANOVA, there was a significant main effect of task (compatible or incompatible) $[F(1,13)=41.99, p<.001]$, indicating that participants were faster for compatible tasks than for incompatible tasks. The main effect of position (one to eight) was also significant $[F(7,91)=16.85, p<.001]$. The interactions between task and position $[F(7,91)=6.40, p<.001]$ and SOA and position $[F(14,182)=7.47, p<.001]$ were significant, but were subsumed in a significant three-way interaction involving task, SOA, and position $[F(14,182)=$ $2.05, p<.05]$. The eight levels involved in the position factor make it difficult to interpret effects involving position without a large number of $t$ tests, so a second ANOVA was carried out on the RTs of Trial 1 (switch) and the mean RT of Trials 4, 5, 6, and 7 (repeat). These results are illustrated in Figure 2. In this analysis, all three main effects were significant: switch-repeat $[F(1,13)=24.52, p<$ $.001]$, revealing reliable switch costs; SOA $(200,550$, and $1,100)[F(2,26)=7.00, p<.005]$, with slowest responses at the 200-msec SOA; and task (compatible or incompatible) $[F(1,13)=16.82, p<.005]$, with faster responses for the compatible task. All three potential interactions were also significant: between SOA and switch-repeat $[F(2,26)=18.09, p<.001]$, between task and switchrepeat $[F(1,13)=12.75, p<.005]$, and between task and SOA $[F(2,26)=4.09, p<.05]$. In this analysis, these two-way interactions were again subsumed by a three-way interaction of SOA, task, and position that was very nearly significant $[F(2,26)=3.23, p=.056]$. The most succinct interpretation of this interaction is that there were significant switch costs across all SOAs for the compatible task, but that switch costs were only significant for the incompatible task at the shortest SOAs (see Figure 2).

\section{Errors}

The only significant main effect was that of position $[F(7,91)=3.20, p<.05]$. The interaction between task and position was also significant $[F(7,91)=2.74, p<$ .05]. To mirror the RT analysis, the accuracy of Trial 1 (switch) and the mean accuracy of Trials 4, 5, 6, and 7 (no switch) were submitted to an ANOVA. There was a significant main effect of switch-no switch $[F(1,13)=5.51$, $p<.05]$, revealing reliable switch costs (see Figure 2). In addition, the interaction between switch-repeat and task was again significant $[F(1,13)=6.68, p<.05]$, reflecting the fact that there were significant switch costs in the compatible condition $[t(13)=2.88, p<.05]$, but not in 


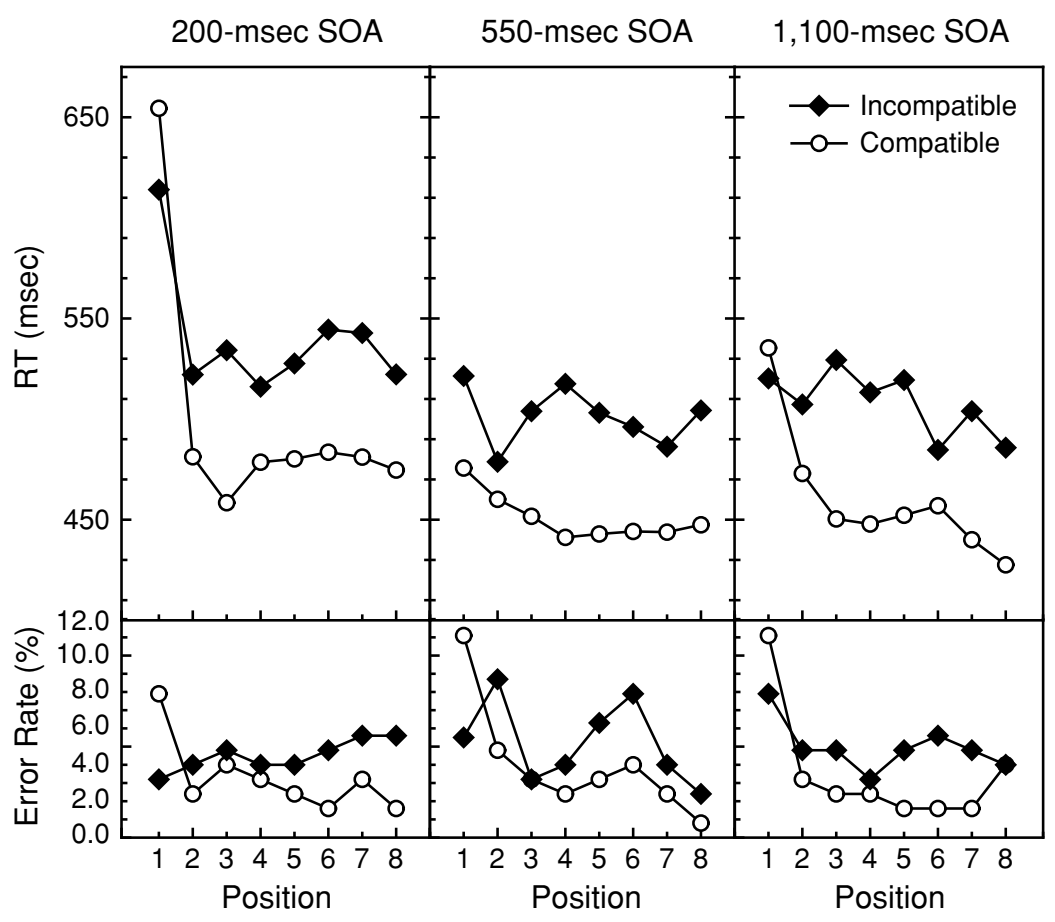

Figure 1. Reaction time (RT; top panels) and error rate (bottom panels) are shown as a function of the trial's position in a run of eight trials following a change in task. Participants switched between making compatible (respond with vibrated finger) and incompatible (respond with nonvibrated finger) responses to a light finger vibration. The three preparatory intervals $(200,550$, or 1,100 msec) were blocked.

the incompatible condition $[t(13)=0.39]$. No other main effects or interactions were significant.

\section{DISCUSSION}

The tactile-manual task used in the present study is like the saccade task in Hunt and Klein (2002), in that making manual responses to tactile stimulation can be classified as a hypercompatible task (Leonard, 1959). On the assumption that hypercompatibility is an important feature for eliminating switch costs, we predicted that the present results would mirror those of the saccade task in Hunt and Klein (2002, Experiment 1). If instead there is something specific about eye movements that is not captured by the tactile hypercompatible task, the results should mirror those of the visual-manual RT task used in Experiment 2 of our 2002 study.

The results are only partially consistent with the hypercompatibility explanation for residual switch costs. Our original hypothesis was that the imperative stimulus, when presented, elicits the automatic retrieval of the response rule used on the previous trial. When the task repeats, this retrieval process produces faster and more accurate responses, but when the task switches, it causes interference with the correct response. In the case of a hypercompatible task, the response is so efficient and automatic that it bypasses the response retrieval process, thus producing no benefit on task repetition trials and no inter- ference when the task switches to an incompatible task, because the simplicity of the preceding task did not leave a memory trace powerful enough to cause measurable response interference (for a more detailed explanation of this model, see Hunt \& Klein, 2002). Consistent with this prediction, in the present study we observed a lack of an interference for switching from compatible to incompatible tasks, but we also observed consistent interference for switching from an incompatible task back to a compatible task, even at the longest SOA. ${ }^{3}$ The latter finding is at odds with results obtained in the saccade task, suggesting that there is something unique about eye movement responses, and how they are affected by a change in task, that is not captured by the tactile choice reaction task used in the present study. Other recent studies of task switching using eye movement tasks have shown the same elimination of residual switch costs (Hodgson, Golding, Molyva, Rosenthal, \& Kennard, 2004; Manoach et al., 2002), but a total elimination of switch costs has yet to be observed in a task that does not involve eye movements, suggesting it to be a unique feature of this response system.

Another unique feature of saccadic task switching is the paradoxical switch benefit seen among antisaccades, with faster and more accurate antisaccade performance on the first trial after a switch from prosaccades (Cherkasova, Manoach, Intriligator, \& Barton, 2002; Hunt \& Klein, 2002). Barton, Greenzang, Hefter, Edelman, and Manoach (2006) have suggested that this benefit reflects 

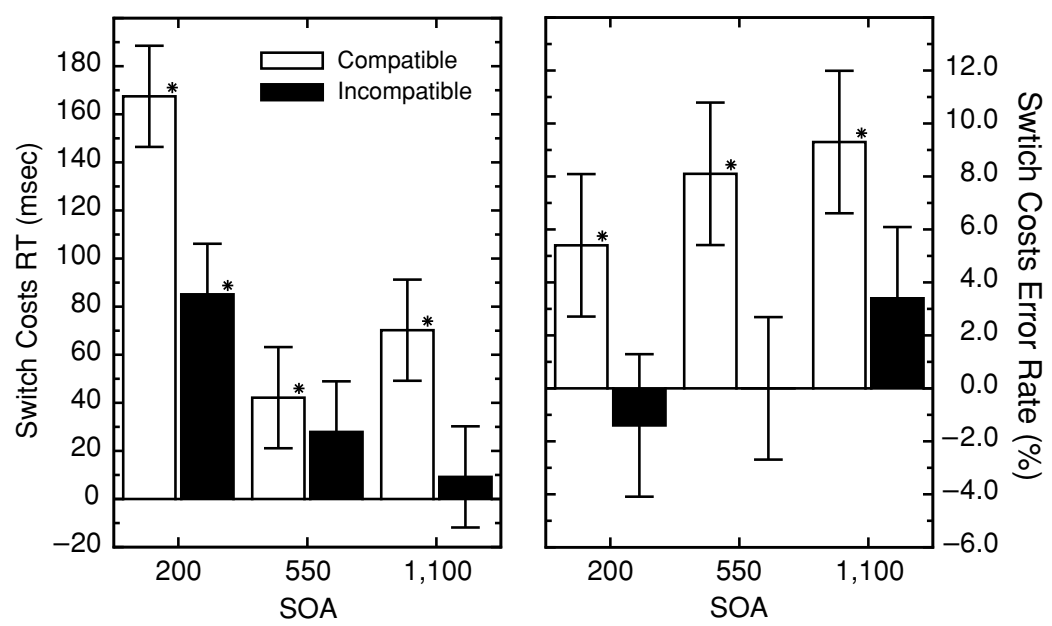

Figure 2. From left to right: Switch costs in RT (in milliseconds) and switch costs in accuracy (\%) as a function of stimulus onset asynchrony. Switch costs are represented by the difference between Trial 1 (the first trial of a new task) and the mean of Trials $4,5,6$, and 7. Positive bars indicate a cost, and negative bars a benefit, associated with switching tasks. The error bars are calculated according to the methods recommended by Masson and Loftus (2003) and Masson (2004) for depicting $95 \%$ confidence intervals in within-subjects experimental designs. " $S$ witch costs are significant.

the suppression of the saccade system necessary for successful antisaccade performance, which carries over from one task to the next, so that any trial preceded by an antisaccade will be slower and any trial preceded by a prosaccade will be faster. Barton et al. proposed that this interpretation can also apply to switch costs in other asymmetric task pairings in which a prepotent response must be suppressed. However, the fact that no switch benefit was observed for the incompatible manual task in both the present study and in Hunt and Klein (2002) suggests that this switch benefit is in fact specific to saccades, because it was not observed in these two otherwise very similar tasks that do not involve saccade responses.

The present results do reveal an asymmetry in switch costs, however, with the more prepotent compatible response showing larger switch costs than those for responses on the incompatible task. Asymmetry of this type is a common feature in task-switching research (e.g., Allport et al., 1994; Wylie \& Allport, 2000) and has been interpreted as evidence of proactive interference from the previous task to the current one, which is greatest when the previous task requires a greater degree of control to perform. However, Yeung and Monsell (2003) have shown that these asymmetries occur only when the responses between the two tasks overlap perfectly and when there is a large degree of interference between the tasks. Under other conditions, switch cost asymmetries can be eliminated or even reversed. In the above study, Yeung and Monsell argued that there is flexibility in the control over the activation level of the current task that can strengthen or weaken task priming effects (i.e., the increase in performance efficiency with task repetitions). One puzzle that remains to be solved is how paradoxical switch cost benefits seen in antisac- cade performance can be explained through a flexiblecontrol model of task priming effects.

A possible explanation for the unique results observed when task switching is measured using saccadic responses is the direct connection between the retina and the superior colliculus, a midbrain structure central to the control of reflexive eye movements. This pathway, which bypasses the cerebral cortex entirely, is likely involved in the control of prosaccades, and it could support a response that does not involve episodic retrieval processes. In other words, this system may be more hypercompatible than other kinds of habitual or easily learned responses, and the otherwise comparable tactile-manual responses do not bypass the response retrieval stage to the same extent as prosaccadic responses. Another consideration is the close alignment of the sensory and motor maps within the superior colliculus and in the frontal eye fields, which support cortically controlled eye movements. The unique architecture of the eye movement system supports a very fast translation of sensory input into a motor response that need not involve any complex response selection processes.

A final caveat is that several distinct types of task switching exist in the literature. For instance, there is a cost of alternating between two tasks relative to performing a single task (see, e.g., Allport et al., 1994), a cost of voluntary switches in task (see, e.g., Arrington \& Logan, 2004), and a cost of switching between runs of repeating tasks (see, e.g., Rogers \& Monsell, 1995). The latter type of task switching was used in the present study, in which switch trials were compared with repeat trials that reached an asymptotic level of performance within the run of trials. Performance on the repeat trials here is not quite the same as performance on the same trial within a block in which the other task is never 
performed (e.g., like those used by Wylie \& Allport, 2002). One of the important debates in the literature is how various task-switching designs might contribute to the switch cost effect (see, e.g., Altmann, 2004). Future research is needed so that the present study's conclusions can be applied to other types of task switching, and in order to understand the relationships between these various kinds of task switching more generally.

\section{REFERENCES}

Allport, D. A., Styles, E. A., \& Hsieh, S. (1994). Shifting intentional set: Exploring the dynamic control of tasks. In C. Umiltà \& M. Moscovitch (Eds.), Attention and performance XV: Conscious and nonconscious information processing (pp. 421-452). Cambridge, MA: MIT Press, Bradford Books.

Altmann, E. M. (2004). Advance preparation in task switching: What work is being done? Psychological Science, 15, 616-622.

Arrington, C. M., \& Logan, G. D. (2004). The cost of a voluntary task switch. Psychological Science, 15, 610-615.

Barton, J. J. S., Greenzang, C., Hefter, R., Edelman, J., \& ManoACH, D. S. (2006). Switching, plasticity, and prediction in a saccadic task-switch paradigm. Experimental Brain Research, 168, 76-87.

Cherkasova, M. V., Manoach, D. S., Intriligator, J. M., \& BarTON, J. J. S. (2002). Antisaccades and task-switching: Interactions in controlled processing. Experimental Brain Research, 144, 528-537.

Dassonville, P., Lewis, S. M., Foster, H. E., \& Ashe, J. (1999). Choice and stimulus-response compatibility affect duration of response selection. Cognitive Brain Research, 7, 235-240.

FitTs, P. M., \& Posner, M. I. (1967). Human performance. Belmont, CA: Brooks-Cole.

Greenwald, A. G. (1970). A choice reaction time test of ideomotor theory. Journal of Experimental Psychology, 86, 20-25.

GreENwaLD, A. G. (1972). On doing two things at once: Time sharing as a function of ideomotor compatibility. Journal of Experimental Psychology, 94, 52-57.

Hodgson, T. L., Golding, C., Molyva, D., Rosenthal, C. R., \& Kennard, C. (2004). Eye movements during task switching: Reflexive, symbolic, and affective contributions to response selection. Journal of Cognitive Neuroscience, 16, 318-330.

Hunt, A. R., \& Klein, R. M. (2002). Eliminating the cost of task set reconfiguration. Memory \& Cognition, 30, 529-539.

LEONARD, J. A. (1959). Tactual choice reactions: I. Quarterly Journal of Experimental Psychology, 11, 76-83.

Logan, G. D. (1988). Toward an instance theory of automatization. Psychological Review, 95, 492-527.

Manoach, D. S., Lindgren, K. A., Cherkasova, M. V., Goff, D. C., Halpern, E. F., Intriligator, J., \& Barton, J. J. S. (2002). Schizophrenic subjects show deficient inhibition but intact task switching on saccadic tasks. Biological Psychiatry, 51, 816-826.

Masson, M. E. J. (2004). Correction to Masson and Loftus (2003). Canadian Journal of Experimental Psychology, 58, 289.
Masson, M. E. J., \& Loftus, G. R. (2003). Using confidence intervals for graphically based data interpretation. Canadian Journal of Experimental Psychology, 57, 203-220.

MeIRAN, N. (1996). Reconfiguration of processing mode prior to task performance. Journal of Experimental Psychology: Learning, Memory, \& Cognition, 22, 1423-1442.

Meiran, N. (2000). Modeling cognitive control in task-switching. Psychological Research, 63, 234-249.

Pashler, H., Carrier, M., \& Hoffman, J. (1993). Saccadic eye movements and dual-task interference. Quarterly Journal of Experimental Psychology, 46A, 51-82.

Pashler, H., Jolicceur, P., Dell'Acqua, R., Crebolder, J., GoschKE, T., DE Jong, R., ET AL. (2000). Task switching and multitask performance. In S. Monsell \& J. Driver (Eds.), Control of cognitive processes: Attention and performance XVIII (pp. 275-423). Cambridge, MA: MIT Press.

Rogers, R. D., \& Monsell, S. (1995). Costs of a predictable switch between simple cognitive tasks. Journal of Experimental Psychology: General, 124, 207-231.

Wylie, G., \& AlLPORT, [D.] A. (2000). Task switching and the measurement of "switch costs." Psychological Research, 63, 212-233.

Yeung, N., \& Monsell, S. (2003). Switching between tasks of unequal familiarity: The role of stimulus-attribute and response-set selection. Journal of Experimental Psychology: Human Perception \& Performance, 29, 455-469.

\section{NOTES}

1. We also could have selected a task that was high in what Greenwald $(1970,1972)$ termed "ideomotor-compatibility"- that is, tasks for which the perceptual input and motor output are highly similar, such as repeating a word or mimicking a gesture. These sorts of tasks would also meet the criteria for hypercompatibility.

2. This is the measure of switch costs used by Hunt and Klein (2002), applied here as well to ease comparison across studies. It is important to note, however, that when the switch trial is removed from the analysis of the present results, there is no longer any effect of sequence, and there are no interactions involving sequence, suggesting that whichever trials were selected for comparison against the switch trials, the results of the analysis would be equivalent.

3. It is yet possible that the small and nonsignificant switch costs to RT and accuracy in the incompatible condition might have been significant had there been more power to detect them, so the possibility remains that the tactile-manual task switch costs do in fact produce the same pattern of results as did the visual-manual task in Hunt and Klein (2002, Experiment 2). In both cases, there appears to have been a gradual change in the speed-accuracy trade-off function, whereby participants tended to be slow and accurate on switch trials at short SOAs, but fast and less accurate at longer SOAs. Although not actually significant in the present study, this is an interesting trend that warrants further investigation.

(Manuscript received November 8, 2004; revision accepted for publication March 24, 2006.) 